
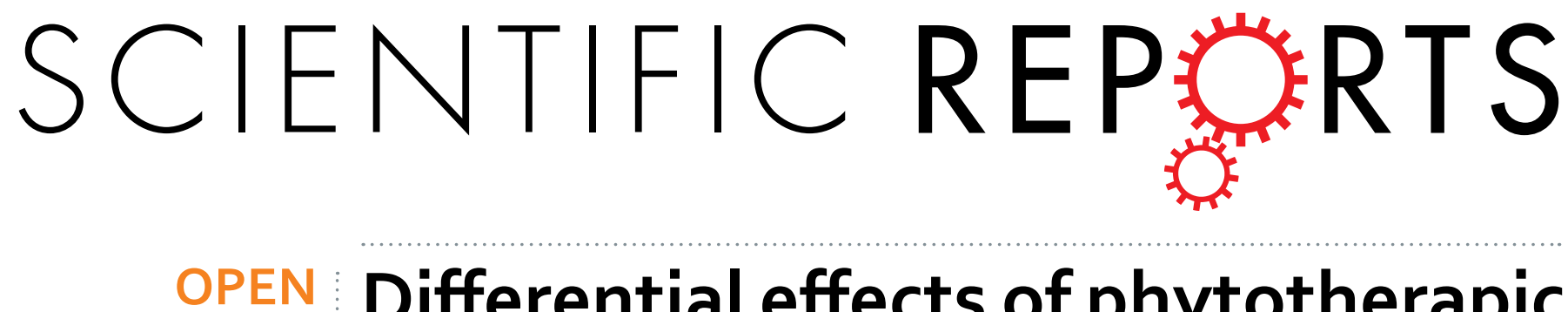

\title{
Differential effects of phytotherapic preparations in the hSOD1 Drosophila melanogaster model of
}

Received: 03 October 2016

Accepted: 15 December 2016

Published: 19 January 2017

\section{ALS}

Francescaelena De Rose ${ }^{1, *}$, Roberto Marotta ${ }^{2, *}$, Giuseppe Talani ${ }^{3, *}$, Tiziano Catelani ${ }^{2}$, Paolo Solari ${ }^{1}$, Simone Poddighe ${ }^{4}$, Giuseppe Borghero ${ }^{4}$, Francesco Marrosu ${ }^{4}$, Enrico Sanna ${ }^{5}$, Sanjay Kasture ${ }^{6}$, Elio Acquas $^{5}$ \& Anna Liscia ${ }^{1}$

The present study was aimed at characterizing the effects of Withania somnifera (Wse) and Mucuna pruriens (Mpe) on a Drosophila melanogaster model for Amyotrophic Lateral Sclerosis (ALS). In particular, the effects of Wse and Mpe were assessed following feeding the flies selectively overexpressing the wild human copper, zinc-superoxide dismutase (hSOD1-gain-of-function) in Drosophila motoneurons. Although ALS-hSOD1 mutants showed no impairment in life span, with respect to GAL4 controls, the results revealed impairment of climbing behaviour, muscle electrophysiological parameters (latency and amplitude of ePSPs) as well as thoracic ganglia mitochondrial functions. Interestingly, Wse treatment significantly increased lifespan of hSDO1 while Mpe had not effect. Conversely, both Wse and Mpe significantly rescued climbing impairment, and also latency and amplitude of ePSPs as well as failure responses to high frequency DLM stimulation. Finally, mitochondrial alterations were any more present in Wse- but not in Mpe-treated hSOD1 mutants. Hence, given the role of inflammation in the development of ALS, the high translational impact of the model, the known anti-inflammatory properties of these extracts, and the viability of their clinical use, these results suggest that the application of Wse and Mpe might represent a valuable pharmacological strategy to counteract the progression of ALS and related symptoms.

Amyotrophic lateral sclerosis (ALS) is a neurodegenerative disorder characterized by the progressive loss of brain and spinal cord motoneurons (MNs). This affects the voluntary movements and relentlessly leads to paralysis of the whole body and finally death within 3 to 5 years of onset. Approximately $10 \%$ of ALS cases are inherited and around $20 \%$ of these familial cases are linked to mutations in the gene encoding the enzyme $\mathrm{Cu} / \mathrm{Zn}$ superoxide dismutase 1 (SOD1) $)^{1,2}$. SOD1 is ubiquitously expressed in all cells and its best characterized function is the dismutation of the highly toxic superoxide anion radical into molecular oxygen and hydrogen peroxide, thus providing a defence against reactive oxygen species toxicity. To date about 150 ALS associated mutations in SOD1 gene have been identified, the majority of which are missense point mutations. Mutated SOD1 gene can acquire both gain and loss of function. In mice, lack of SOD 1 function does not lead to development of neurodegeneration, while its over-expression leads to ALS symptoms ${ }^{3-5}$. This evidence suggest that the toxicity related to these mutations depends on a toxic gain of function (GOF) rather than on a loss of function (LOF). The cellular and molecular mechanism through which these mutations (mutant SOD1) induce onset and progressive spreading of ALS pathology is complex and still not fully understood. This mechanism includes oxidative stress, mitochondrial damage, axonal transport impairment, glutamatergic excito-toxicity, activation of endoplasmic reticulum stress, RNA metabolism impairment, interaction with glial cells ${ }^{6}$ and protein aggregation ${ }^{7}$. In fact, SOD1 mutant proteins tend to be misfolded and form protease-resistant aggregates causing death of motoneurons ${ }^{8}$. Mutant

${ }^{1}$ University of Cagliari, Department of Biomedical Sciences, Monserrato, 09042, Italy. ${ }^{2}$ Istituto Italiano di Tecnologia (IIT), Department of Nanochemistry, Genova, 16163, Italy. ${ }^{3}$ National Research Council (CNR), Institute of Neuroscience, Monserrato, 09042, Italy. ${ }^{4}$ University of Cagliari, Department of Public Health, Clinical and Molecular Medicine, Monserrato, 09042, Italy. ${ }^{5}$ University of Cagliari, Department of Life and Environmental Sciences, Cagliari, 09124, Italy. ${ }^{6}$ Pinnacle Biomedical Research Institute, Bhopal, 462003, India. *These authors contributed equally to this work. Correspondence and requests for materials should be addressed to A.L. (email: liscia@unica.it) 
SOD1 can seed misfolding and aggregation of endogenous wild-type SOD1 and transfer from cell to cell causing the intercellular transmission of disease through the central nervous system, a propagation mechanism similar to prion replication and spreading ${ }^{9,10}$.

It is worth noting that both insects and human beings share a number of genes and the organization and cellular function of the nervous system, making the Drosophila melanogaster a useful and powerful model for understanding the biological bases of human pathology and gaining evidence of high translational significance of such model and its potential therapeutic effectiveness. Accordingly, a number of neurodegenerative diseases, including Alzheimer's ${ }^{11-13}$ and Parkinson's disease ${ }^{14-19}$ as well as ALS, object of this study ${ }^{20,21}$, are heuristically modeled in Drosophila. However, despite the significant progress in the knowledge of the biological bases of the degenerative diseases, the hope to find an efficient treatment has been, so far, elusive, as no treatment can halt their progression, with the partial exception of the antiglutamatergic Riluzole ${ }^{\circledR}$.

In this context, given the results obtained in two different Drosophila models of Parkinson disease (PD-LRRK2-LOF and PD-PINK1 ${ }^{\mathrm{B} 9}$-LOF) with the application of two methanolic extracts of parts of the medicinal plants, Withania somnifera and Mucuna pruriens widely used in the Ayurvedic medicine ${ }^{17-19,22-24}$ for their potential effects on treating many central nervous system disorders, we deemed to investigate their effects on ALS symptoms. In order to obtain experimental data, we proceed to set up a neurophysiological, biochemical and histological investigation using the hSOD1-GOF Drosophila model of ALS. Thus, based on the observations (1) that the PD-LRRK2 and ALS-SOD1 diseases share common oxidative stress- and neuroinflammation-based mechanisms and (2) that both Withania somnifera and Mucuna pruriens are endowed with potent anti-oxidative and anti-inflammatory properties, the results of the present study are expected to provide new data on the pathophysiology of ALS models and to suggest possible new vistas in the inflammatory, oxidative stress, genetic roles correlated to this neurodegeneration. In particular, given that neurodegenerative diseases are often associated with abnormal protein accumulation ${ }^{25,26}$, the mechanism by which ALS may take place is by the overexpression of human SOD1 in the motoneurons in agreement with Stathopulos et al. ${ }^{27}$ that could be regarded as a destabilizing solution condition resulting as a consequence in loss of mitochondrial functionality and also in altered vescicle trafficking. Thus, in particular, Mpe treatment might enhance bruchpilot synthesis similarly to what we found in another neurodegenerative disease in PD-PINK $1{ }^{\mathrm{B} 9}$ Drosophila mutants ${ }^{18}$, while Wse treatment might act by enhancing the vesicle trafficking as previously suggested in LRRK2 ${ }^{19}$. Moreover, in the context of current knowledge about the mechanism of action of these drugs, even if they do not specifically regard ALS, a few references provide insight on the mechanism by which these compounds exert their anti-inflammatory effects ${ }^{28,29}$.

\section{Results}

Wse, but not Mpe, treatment enhances survival rate of hSOD1 flies. Figure la shows that the mutant hSOD1 that over expressed human SOD1 in the motoneurons exhibited no significant change in lifespan compared to GAL4. Accordingly to previous results, obtained with fly models of Parkinson's disease ${ }^{18,19}$, the hSOD1 mutants were treated with $W s e$ or $M p e$ at $0.1 \% \mathrm{w} / \mathrm{w}$ in their standard diet starting from larvae stage $\left(\mathrm{L}^{+} / \mathrm{A}^{+}\right)$or as adults only $\left(\mathrm{L}^{-} / \mathrm{A}^{+}\right)$. As shown by Kaplan-Meier survival curves, administration of $W s e$ as $\mathrm{L}^{-} / \mathrm{A}^{+}$ exerts great significant effects in mutant flies (Fig. 1b) with a significant increase in lifespan compared to not treated mutants. Surprisingly, the average lifespan of Wse-treated hSOD1 mutants was also longer than that of GAL4 (being 50\% and 58\% of survival at 40-days-old in GAL4 and in Wse-treated hSOD1 mutants, respectively). In contrast, $M p e$ administered as $\mathrm{L}^{-} / \mathrm{A}^{+}$had no effect on the lifespan of mutant hSOD1 (Fig. 1c) ( $\mathrm{p}>0.05$ at Gehan-Breslow-Wilcoxon test). As expected, neither Wse nor Mpe affected the lifespan of the GAL4 control flies (Fig. 1d). In addition, when administered as $\mathrm{L}^{+} / \mathrm{A}^{+}$, a dramatic decrease of the lifespan was induced by both Wse or Mpe (Fig. 1b,c).

Wse and Mpe treatment ameliorates the climbing behaviour of hSOD1 flies. As shown in Fig. 2a, hSOD1 mutants showed a significant increase in the climbing time in groups I and II compared to the GAL4 line, with a worsening trend with aging displayed at age stage III in which also GAL4 showed a significant slowing-down of climbing activity. Notably, flies overexpressing hSOD1 in motoneurons showed a significant impairment of climbing activity within the first week (age group I shown in Fig. 2) when compared with flies expressing dSOD1 i.e. GAL4. In order to further characterize the effects of the extracts on this genetic model of ALS, we tested climbing activity of flies administered with Wse or Mpe (Fig. 2a,b) (as $\left.\mathrm{L}^{-} / \mathrm{A}^{+}\right)$. Administration of Wse or Mpe was done in these experiments only in the condition $\mathrm{L}^{-} / \mathrm{A}^{+}$, suggested by the lifespan experiments which demonstrated that the administration to flies as $\mathrm{L}^{+} / \mathrm{A}^{+}$resulted in a dramatic decrease of lifespan. Treatment of groups I-III of hSOD1 mutants with both Wse or Mpe $(0.1 \% \mathrm{w} / \mathrm{w})$ induced a recovery of motor disability compared to untreated mutants. Moreover, the percentage of flies that achieved the target (10 s) was very close to $100 \%$ in both Wse- and Mpe-treated flies, higher than those of untreated mutants and, surprisingly, also in respect to age-group III GAL4 flies (Fig. 2a,b).

Wse and Mpe affect kinetic properties of evoked PSPs recorded from DLM in hSOD1 mutant flies. In these experiments we first evaluated the potential changes in the function of the DLM neuromuscular junction of hSOD1 (group II) compared with GAL4 control flies. Basal kinetic properties (amplitude and latency) of evoked PSPs (ePSPs) recorded from the DLM after Giant Fibre System (GFS) electrical stimulation were analyzed. ePSPs recorded from DLM muscle of GAL4 animals had an average amplitude of $30.98 \pm 3 \mathrm{mV}$ and a latency of $0.92 \pm 0.1 \mathrm{~ms}$ (Fig. 3a-c). Responses measured in hSOD1 mutants were characterized by a significant decrease in amplitude $[20.32 \pm 2.7 \mathrm{mV}$, one-way ANOVA, $\mathrm{F}(3,61)=3.71, \mathrm{P}<0.05$ vs GAL4 Bonferroni’s post-hoc] and a significant increase in latency $[1.4 \pm 1.1 \mathrm{~ms}, \mathrm{~F}(3,61)=5.57, \mathrm{P}<0.01$ vs GAL4 Bonferroni's post-hoc] (Fig. 3a-c), suggesting that this mutation hampers the function of GFS-DLM muscle conduction. Interestingly, treatment of hSOD1 flies with Wse or Mpe reverted the reduction in ePSP amplitude as well as the 


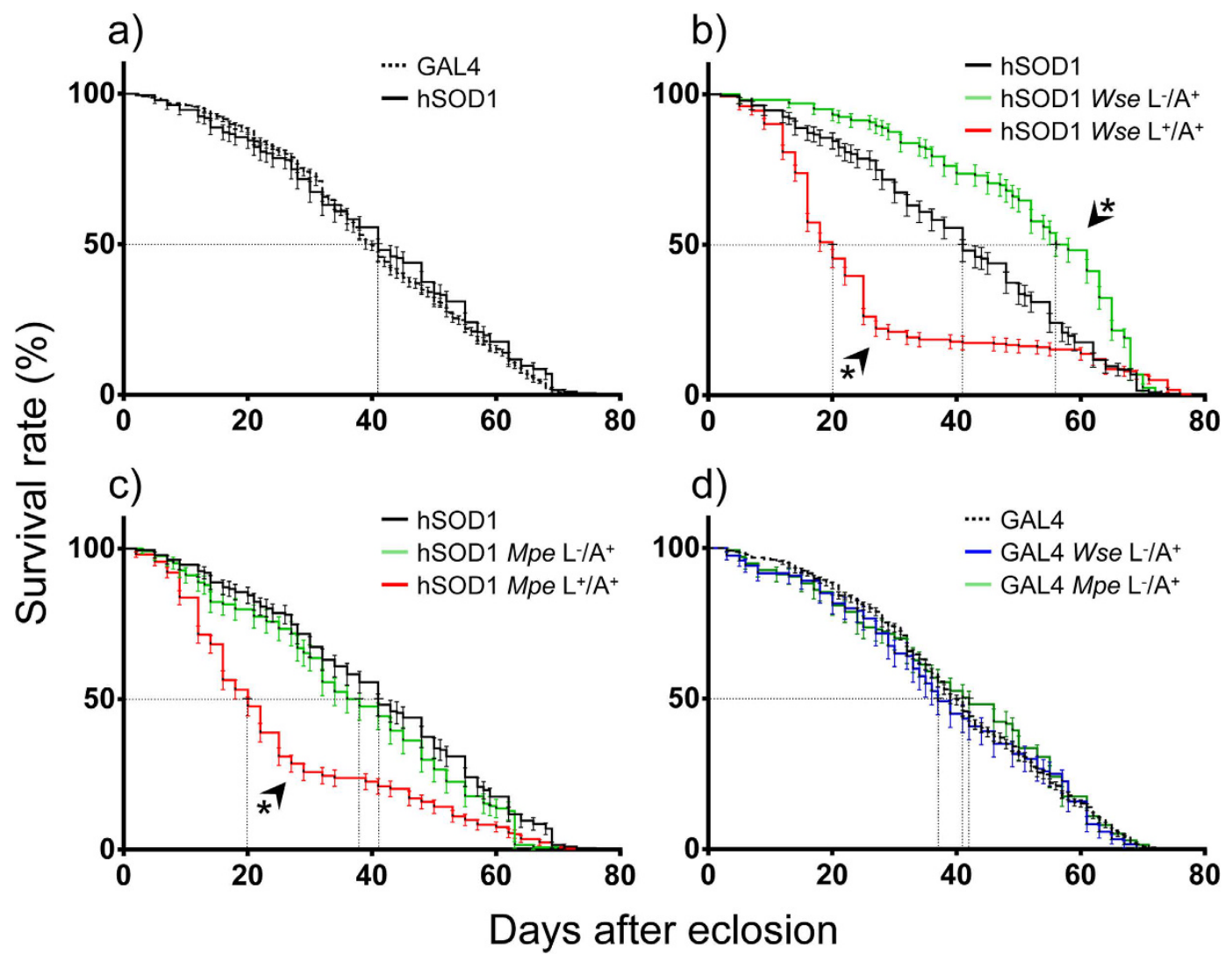

Figure 1. Effects of Withania somnifera extract (Wse) and Mucuna pruriens extract (Mpe) treatment on lifespan. (a) Lifespan, expressed as survival rate (\%), of untreated isogenic flies (Gal4) and untreated mutant flies (hSOD1). (b) Lifespan of untreated hSOD1 compared to Wse-treated hSOD1, only when adults $\left(\mathrm{L}^{-} / \mathrm{A}^{+}\right)$ and from their larval stage to the end of their life cycle $\left(\mathrm{L}^{+} / \mathrm{A}^{+}\right)$. (c) Lifespan of untreated hSOD1 compared to Mpe-treated hSOD1, only when adults $\left(\mathrm{L}^{-} / \mathrm{A}^{+}\right)$and from their larval stage to the end of their life cycle $\left(\mathrm{L}^{+} / \mathrm{A}^{+}\right)$. (d) Lifespan of untreated GAL4 control flies compared with Wse- and Mpe- treated flies only when adults $\left(\mathrm{L}^{-} / \mathrm{A}^{+}\right)$. ${ }^{*}$ Indicates $\mathrm{p}<0.05$ at Kaplan-Meier survival curves (Gehan-Breslow-Wilcoxon-Graph Pad Prism 5.01), (b) untreated hSOD1 compared to $\left(\mathrm{L}^{-} / \mathrm{A}^{+}\right)$and $\left(\mathrm{L}^{+} / \mathrm{A}^{+}\right)$Wse-treated hSOD1 and (c) untreated hSOD1 compared to $\left(\mathrm{L}^{+} / \mathrm{A}^{+}\right)$Mpe-treated hSOD1.

increase in latency with values that were not significantly different to those observed in GAL4 flies $(\mathrm{P}>0.05$, one-way ANOVA, Bonferroni's post-hoc) (Fig. 3a,c).

Wse and Mpe ameliorate the ePSP responses to increasing stimulation frequency of GFS in hSOD1 mutant flies. We further tested flies, from the different experimental groups, by recording the "frequency of following" which consisted in applying a train of 10 stimuli at different frequencies (from 10 to $200 \mathrm{~Hz}$, with steps of $25 \mathrm{~Hz}$ ) to GFS. As previously reported ${ }^{19,21,30}$, in control flies the number of failures increased depending on the increase in simulation frequency. In particular, in GAL4 flies a train of 10 stimulations delivered at $100 \mathrm{~Hz}$ induced a limited rate of failures $(8.3 \pm 5.6 \%)$, while a train at $200 \mathrm{~Hz}$ resulted in a predictive and consistent increase in failures percentage (27.5 $\pm 8.9^{*} \%, \mathrm{P}<0.05$, one-way ANOVA, Bonferroni's post-hoc) (Fig. 4a,b,d). Interestingly, when hSOD1 flies were exposed to the same protocol, the rate of failures at both $100 \mathrm{and} 200 \mathrm{~Hz}$ resulted significantly enhanced with respect to GAL4 flies $[\mathrm{F}(2,41)=6.8, \mathrm{P}<0.05$, two-way ANOVA Bonferroni's post-hoc] (Fig. 4a,b,d). Our findings are also consistent with behavioural data where hSOD1 mutant flies showed uncoordinated movements impairments that, in turn, could be related to the low probability of muscle contraction. As observed for the basal properties of ePSP, treatment with either Wse or Mpe was able to antagonize the increased rate of failures in response to train stimulations and the percentage of failures observed at $100 \mathrm{~Hz}$ showed values not significantly different from those observed in GAL4 flies (Fig. 4a-e). Surprisingly, both Wse and $M p e$ treatment were able to prevent the effect of the mutation making the response latencies and amplitude recorded in hSOD1-treated flies indistinguishable from those in the GAL4 flies.

Interestingly treatment with Wse and Mpe in GAL4 animals failed to change both sPSP amplitude and latency when compared with untreated GAL4 animals (one-way ANOVA, $\mathrm{F}(2,24)=1.19, \mathrm{P}>0.05$ vs GAL4 Bonferroni's post-hoc) (Fig. 5a,b). Moreover, even for "frequency of following" responses, treatment with Wse and Mpe in GAL4 animals failed to change this response of sPSP when compared with untreated GAL4 animals (Fig. 5c).

Wse, but not Mpe, treatment rescues the impairment of mitochondrial morphology in motoneurons of hSOD1 mutant flies. Transmission (TEM) and scanning transmission electron microscopy 
a)

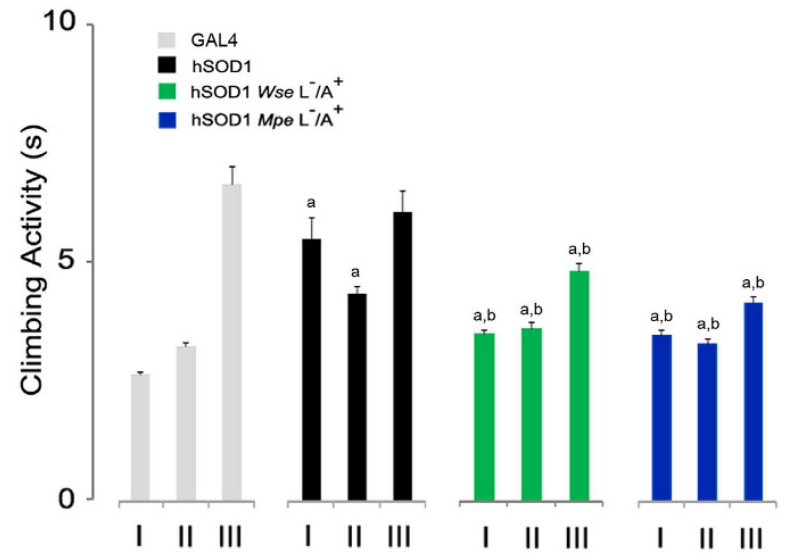

b)

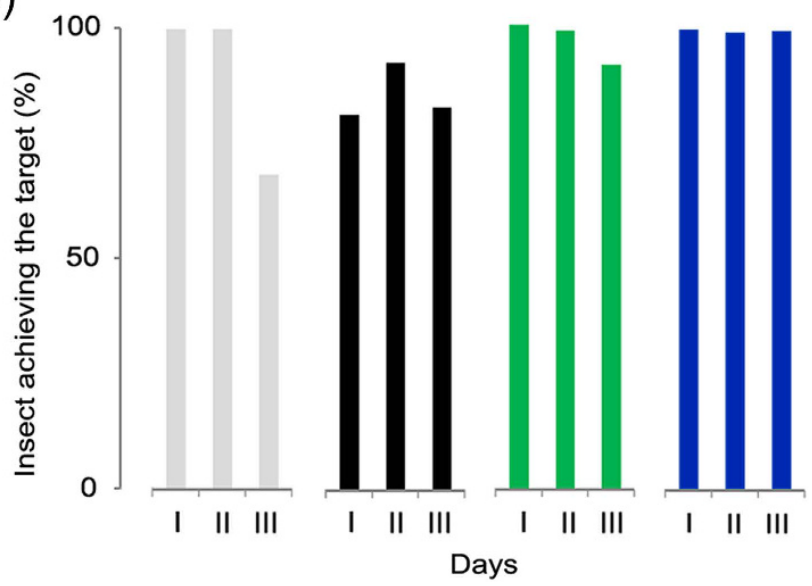

Figure 2. Effects of Wse and Mpe on climbing activity. (a) Climbing activity of adult GAL4 flies, untreated mutants (hSOD1) and Wse- and Mpe-treated hSOD1. The treatment was administered during the adult stage of mutants $\left(\mathrm{L}^{-} / \mathrm{A}^{+}\right)$and its effect was assayed at three different age steps (I: 3-6; II: 10-15; III: 20-25 days) of

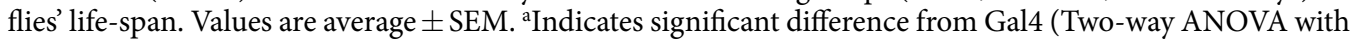
HSD post-hoc test, $\mathrm{p}<0.05$ ); bindicates significant difference from hSOD1 (Two-way ANOVA with HSD posthoc test, $\mathrm{p}<0.05$ ). (b) Percentages of adult Gal4, hSOD1 and $W s e \mathrm{~L}^{-} / \mathrm{A}^{+}$- and $M p e \mathrm{~L}^{-} / \mathrm{A}^{+}$- treated hSOD1 that could climb unto, or above, the line drawn at $6 \mathrm{~cm}$ from the bottom of the tube within 10 seconds.

(STEM) analysis were performed in untreated hSOD1 flies and hSOD1 mutants treated with $0.1 \% \mathrm{w} / \mathrm{w}$ Wse or Mpe as $\mathrm{L}^{-} / \mathrm{A}^{+}$. All flies analyzed were belonging to group II (10-15 days old) at the moment of morphological assessment. A high number of clearly damaged mitochondria with fragmented cristae, abnormally enlarged membranes and inhomogeneous electron transparent matrix have been observed by TEM and EM tomography in the T1- T2 regions of the thoracic ganglia in Drosophila hSOD1 mutants ${ }^{21}$ (Fig. 6a-e and Supplementary Video S1). Intriguingly, a similar density of damaged mitochondria has been observed in those mutants also after treatment with Mpe (Fig. 6c,d). On the contrary Drosophila hSOD1 mutants treated with Wse showed a significant reduction in the number of damaged mitochondria (Fig. 6b,d) compared with untreated hSOD1 flies. The treatment of the hSOD1 mutants with Wse was thus able to significantly decrease the number of damaged mitochondria in T1-T2 regions of thoracic ganglia rescuing also climbing as well as muscle electrophysiological parameters.

\section{Discussion}

In agreement with Watson et al. ${ }^{21}$ the results of the present study disclosed that the survival curves of flies overexpressing hSOD1-GOF in motoneurons were not significantly impaired with respect to GAL4 control flies. As discussed in the review by Casci and Pandey ${ }^{31}$ the overexpression of SOD1 in different tissues may also be responsible of an improvement of life span thus, both the loss of expression of SOD1 and its overexpression may be responsible in affecting the lifespan. Interestingly, administration of Wse or Mpe to hSOD1 mutant flies resulted in differential effects also depending on the maturity stage of the flies. In particular, while both Wse and Mpe administration resulted in a reduced lifespan when administered to flies as $\mathrm{L}^{+} / \mathrm{A}^{+}$, only Wse increased the life duration of hSOD1 mutants when administered as $\mathrm{L}^{-} / \mathrm{A}^{+}$.

Impairment in lifespan following Wse administration to hSOD1 flies as $\mathrm{L}^{+} / \mathrm{A}^{+}$agrees with our previous data regarding the Wse exposure to LRRK2 PD mutants leading us to the conclusions that Wse may have, after long term exposure or exposure at high concentrations, toxic effects ${ }^{19}$. Furthermore, the present observation after Wse 
a)

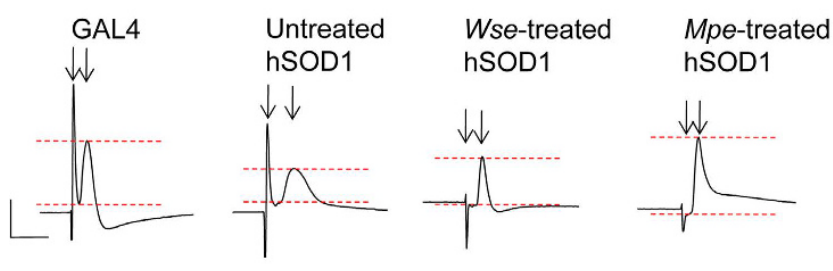

b)

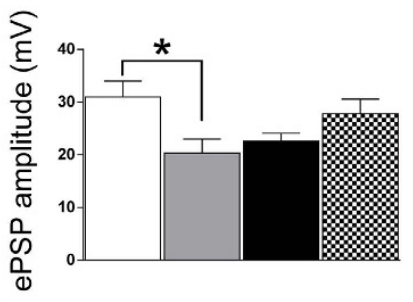

$$
\begin{aligned}
& \square \text { GAL4 } \\
& \square \text { hSOD1 } \\
& \text { Wse-treated hSOD1 } \\
& \text { mos Mpe-treated hSOD1 }
\end{aligned}
$$

c)

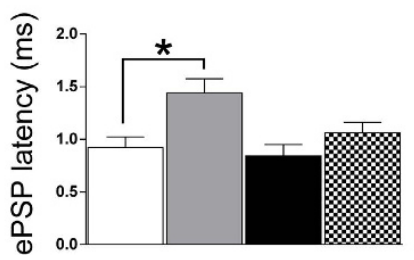

Figure 3. Effect of hSOD1 gene mutation and treatment with Wse or Mpe on ePSP latency and amplitude recorded from Drosophila DLM. (a) Representative traces obtained from four different flies of the different experimental groups in which ePSP latency is calculated as the time (ms) from stimulus application to the peak of PSP (black arrows) and PSP peak is calculated by measuring the maximal amplitude of the response starting from the baseline (red scatter lines). Scale bar, $20 \mathrm{mV} / 5 \mathrm{~ms}$. (b,c) Bar graphs represent the mean $\pm \mathrm{SEM}$ of PSP amplitude $(\mathrm{mV})(\mathbf{b})$ and latency $(\mathrm{ms})$ (c) recorded from flies treated with Wse and with $M p e,{ }^{\star} P<0.05$ compared to GAL4, one-way ANOVA, followed by Bonferroni post-hoc test. N=24 (GAL4), 16 (untreatedhSOD1), 21 (Wse-treated hSOD1) and 16 (Mpe-treated hSOD1).

administration to hSOD1 mutants as $\mathrm{L}^{-} / \mathrm{A}^{+}$appears in agreement with the ability to prolong lifespan of LRRK2 $\mathrm{PD}$ mutants upon treatment as $\mathrm{L}^{-} / \mathrm{A}^{+19}$. This suggests that the widely accepted anti-inflammatory properties of Withania somnifera ${ }^{32}$ may represent the common mechanism underlying lifespan increase of hSOD1 (present data, Fig. 1) (TS50 (untreated hSOD1) $=\sim 20$ days versus TS50 (Wse-treated hSOD1) $=\sim 60$ days) and that of LRRK2 mutants ${ }^{19}$.

Notably, Mpe administration to hSOD1 mutants produced opposite results with respect to our previous study with $M p e$ administration to flies of another genetic model, the PINK $1^{\mathrm{B} 9} \mathrm{PD}$ mutants ${ }^{18}$. In fact, administration of Mpe to PINK1 ${ }^{\mathrm{B} 9} \mathrm{PD}$ mutant flies as $\mathrm{L}^{+} / \mathrm{A}^{+}$resulted in a significant rescue of the lifespan, whereas its administration to $\mathrm{L}^{+} / \mathrm{A}^{+}$hSOD1 resulted in a dramatic lifespan reduction. On the other hand, when Mpe was administered as $\mathrm{L}^{-} / \mathrm{A}^{+}$the extract failed to affect this parameter in both mutants. Anyway, the overall results, suggest that the genetic and metabolic pathways of these two mutants differ significantly as do the effects of these phytotherapic extracts, that, we recall, do not affect the lifespan of GAL4 control flies when administered as $\mathrm{L}^{-} / \mathrm{A}^{+}$.

Also, the observation that both extracts protect against the development of motor impairment suggests that Wse and Mpe can have a positive impact on complex climbing behaviour, although involvement of distinct pathways either in terms of neuronal circuitry and metabolic machinery is still unclear. This seems in agreement also with our previous studies with LRRK2 ${ }^{19}$ and PINK1 $1^{\mathrm{B} 918}$ genetic models of PD in which we found that Wse and Mpe, similarly to the present data at stage II of age, significantly ameliorated climbing behaviour.

Interestingly, both Wse and $M p e$ administered to flies as $\mathrm{L}^{-} / \mathrm{A}^{+}$were able to protect from the climbing impairment in an age-independent manner and, surprisingly, also protected group III hSOD1 mutant flies from the age-related spontaneous impairment as suggested by the observation that we did not detect any significant difference between the dSOD1 (i.e. GAL4) and untreated hSOD1 flies. The observation that in our conditions the motor impairment appears as early as within the first five days (group I) and remains almost constant (Fig. 2), is at variance with what Watson et al. ${ }^{21}$ found in flies expressing WT (not overexpressed) hSOD1 which showed a progressive loss of climbing starting at 21 days as compared with dSOD1 controls. In this respect, the difference we found is likely imputable to differences in the expression level of hSOD1. Accordingly, Watson et al. ${ }^{21}$ reported in Drosophila an accumulation of hSOD1 in motoneurons that increased with the age.

Electrophysiological data showed that hSOD1 over expression in motoneurons was associated with a significant increase in latency of ePSPs with a parallel and significant decrease in amplitude when compared to GAL4 
a) $\begin{array}{lll}\text { GAL4 hSOD1 } & \begin{array}{l}\text { Wse-treated } \\ \text { hSOD1 }\end{array} & \begin{array}{l}\text { Mpe-treated } \\ \text { hSOD1 }\end{array}\end{array}$
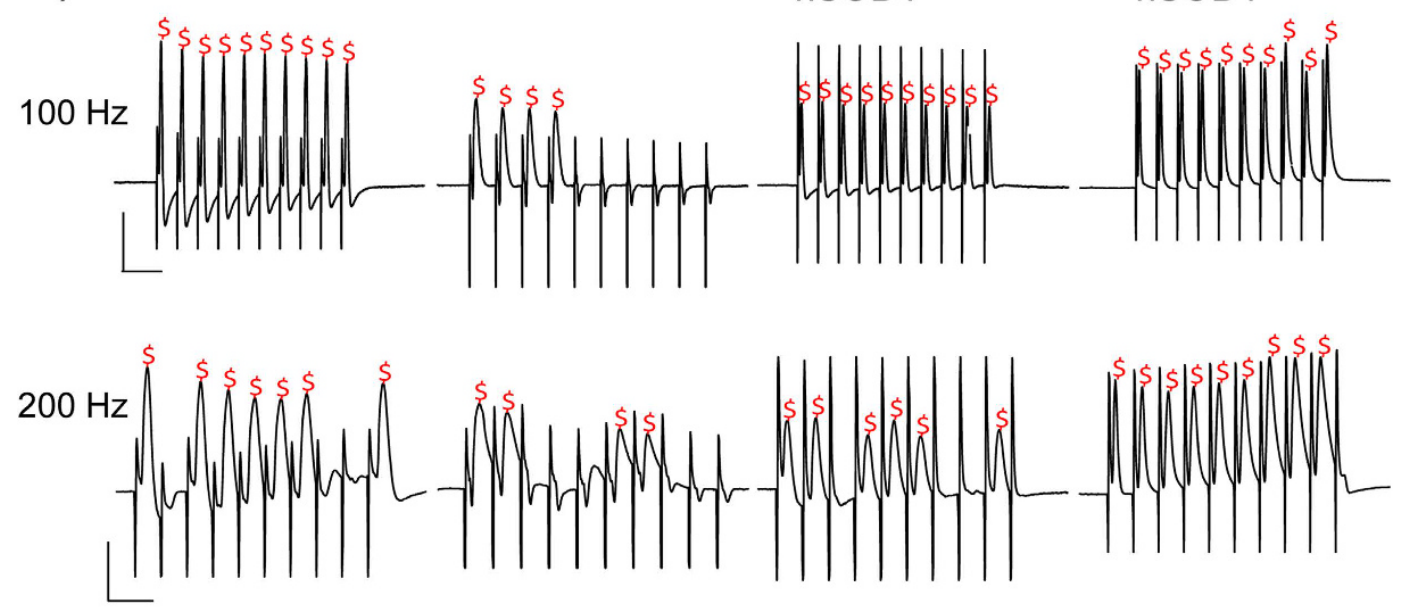

b)

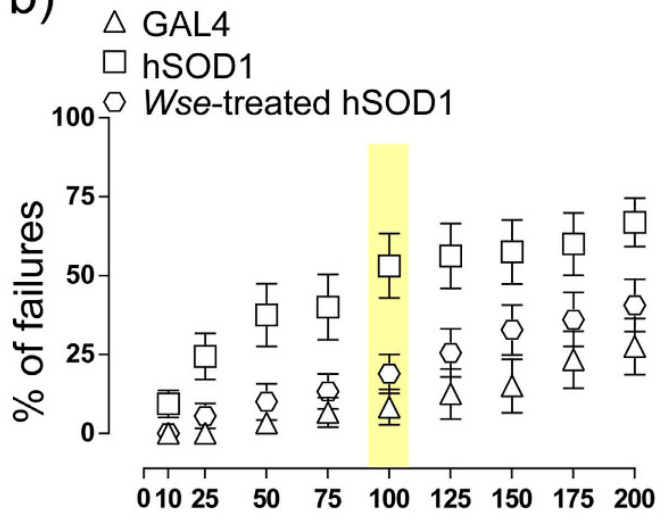

d)

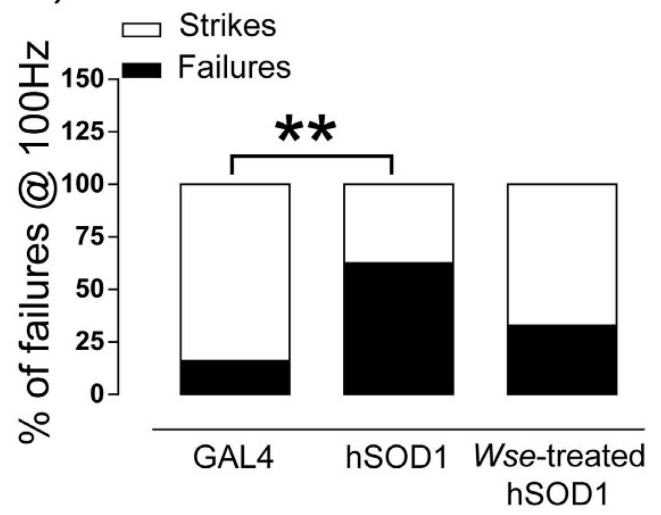

c)

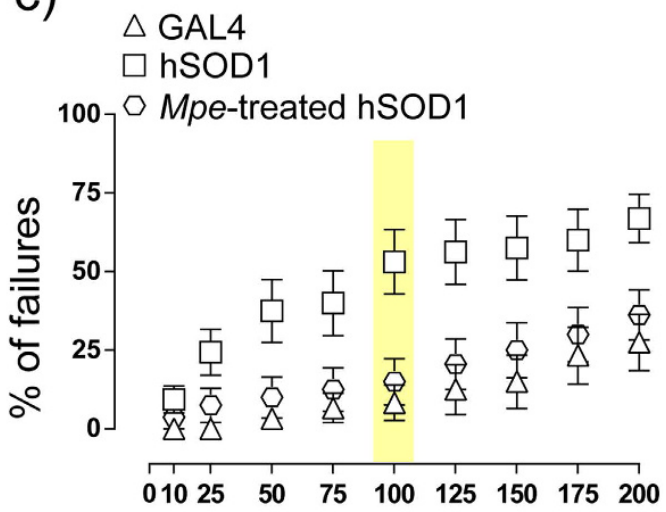

e)

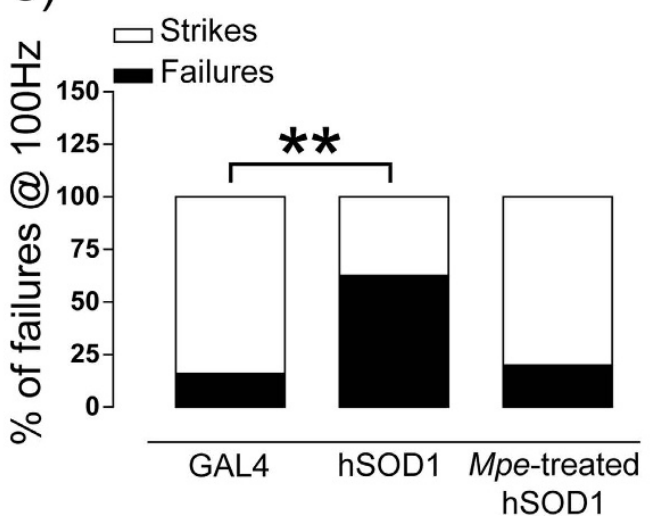

Figure 4. Effect of SOD1 gene mutation and treatment with Wse or Mpe on PSP response to "frequency of following" recorded in Drosophila DLM. (a) Representative traces obtained from four different flies of different experimental group in which PSPs were evoked in response to 10 stimulations at increasing train frequency of 10 consecutive stimuli. ${ }^{\$}$ Indicates the detected response at 100 (top) or $200 \mathrm{~Hz}$ (bottom). Scale bar $20 \mathrm{mV} / 20 \mathrm{~ms}$ for $100 \mathrm{~Hz}$ and $20 \mathrm{mV} / 10 \mathrm{~ms}$ for $200 \mathrm{~Hz}$. (b,d) Scatter plot graphs showing the changes in PSP amplitude following stimulation at increasing frequency (the effect at $100 \mathrm{~Hz}$ is highlighted in yellow). All values are expressed as the mean \pm SEM of the $\%$ of failure observed in every train. In $\mathrm{d}$ the averaged $\%$ of failure was plotted of GAL4, hSOD1 and hSOD1 treated with $W s e .{ }^{*} P<0.01$ compared to GAL4, unpaired t-test. (c,e) Scatter plot graphs showing the changes in PSP amplitude following stimulation at increasing frequency (the effect at $100 \mathrm{~Hz}$ is highlighted in yellow). All values are expressed as the mean $\pm \mathrm{SEM}$ of the $\%$ of failure observed in every train. In e the averaged \% of failure was plotted of GAL4, hSOD1 and hSOD1 treated with Mpe. ${ }^{*} P<0.01$ compared to GAL4, unpaired t-test. 
a)

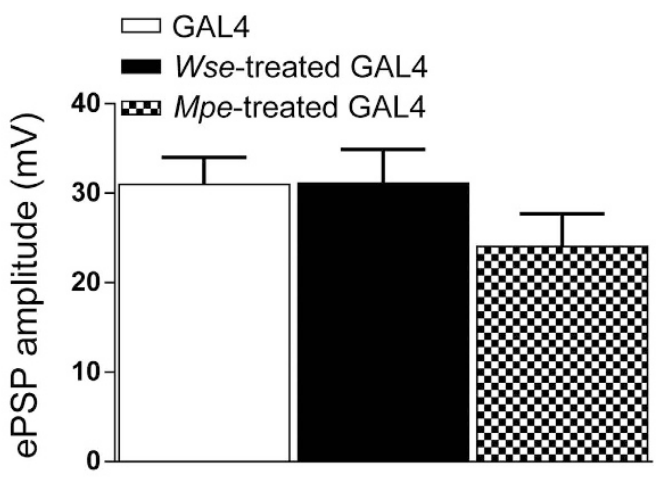

b)

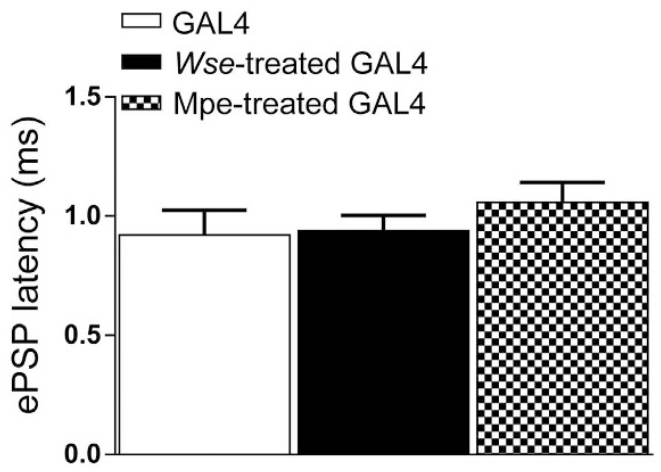

C)

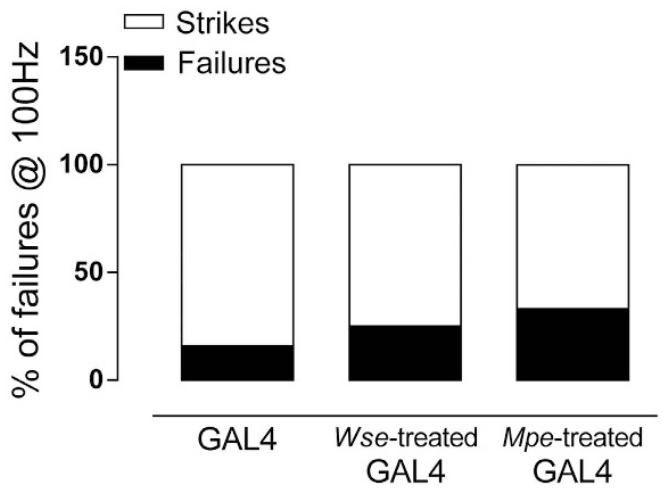

Figure 5. Effects of Withania somnifera extract (Wse) and Mucuna pruriens extract (Mpe) treatment on GAL4 on ePSP latency, amplitude and response to "frequency of following" recorded in Drosophila DLM. $(\mathbf{a}, \mathbf{b})$ Bar graphs, representing the mean \pm SEM of PSP amplitude $(\mathrm{mV})(\mathbf{a})$ and latency $(\mathrm{ms})(\mathbf{b})$ recorded from GAL4 flies and GAL4 treated with Wse or Mpe, indicate that treatment failed to induce change of ePSP parameter when compared with untreated GAL4 $(\mathrm{N}=24$ (GAL4), 8 (Wse-treated GAL4), 7 (Mpe-treated GAL4); (c) Bar graph representing the averaged \% of failure plotted in GAL4 and GAL4 treated with Mpe or Wse, indicate that the treatment fails to alter this parameter when compared with untreated GAL4.

animals, an effect no longer present in hSOD1 flies fed both Wse and Mpe. Conversely, neither Wse nor Mpe affected the GAL4 electrophysiological parameters.

These data are in agreement with previous reports in which hSOD1 flies showed an impairment of synaptic transmission that become progressively defective ${ }^{21}$. Moreover, differently from our data, Watson and colleagues ${ }^{21}$ reported such kind of impairments only in 55 days old flies in contrast to their 10 days old WT hSOD1 flies which showed a normal synaptic response. The differences between the present data and those of Watson et al. ${ }^{21}$ can be attributable to the differences in the genetic background of mutants belonging to these different studies. In fact, in this regard, it should be kept in mind that Watson and colleagues ${ }^{21}$ performed their data on WT hSOD1 or mutation of this gene while we recorded from mutant flies in which hSOD1 was overexpressed and this may lead to a more pronounced impairment in motor neurons, the only cells in which the mutation occur, that may appear earlier in life than in Watson's flies.

The lower amplitude and the higher latency of ePSP shown by hSOD1 muscle responses may be related to a decrease in neuronal conduction and a possible related decrease in the probability of neurotransmitter release 

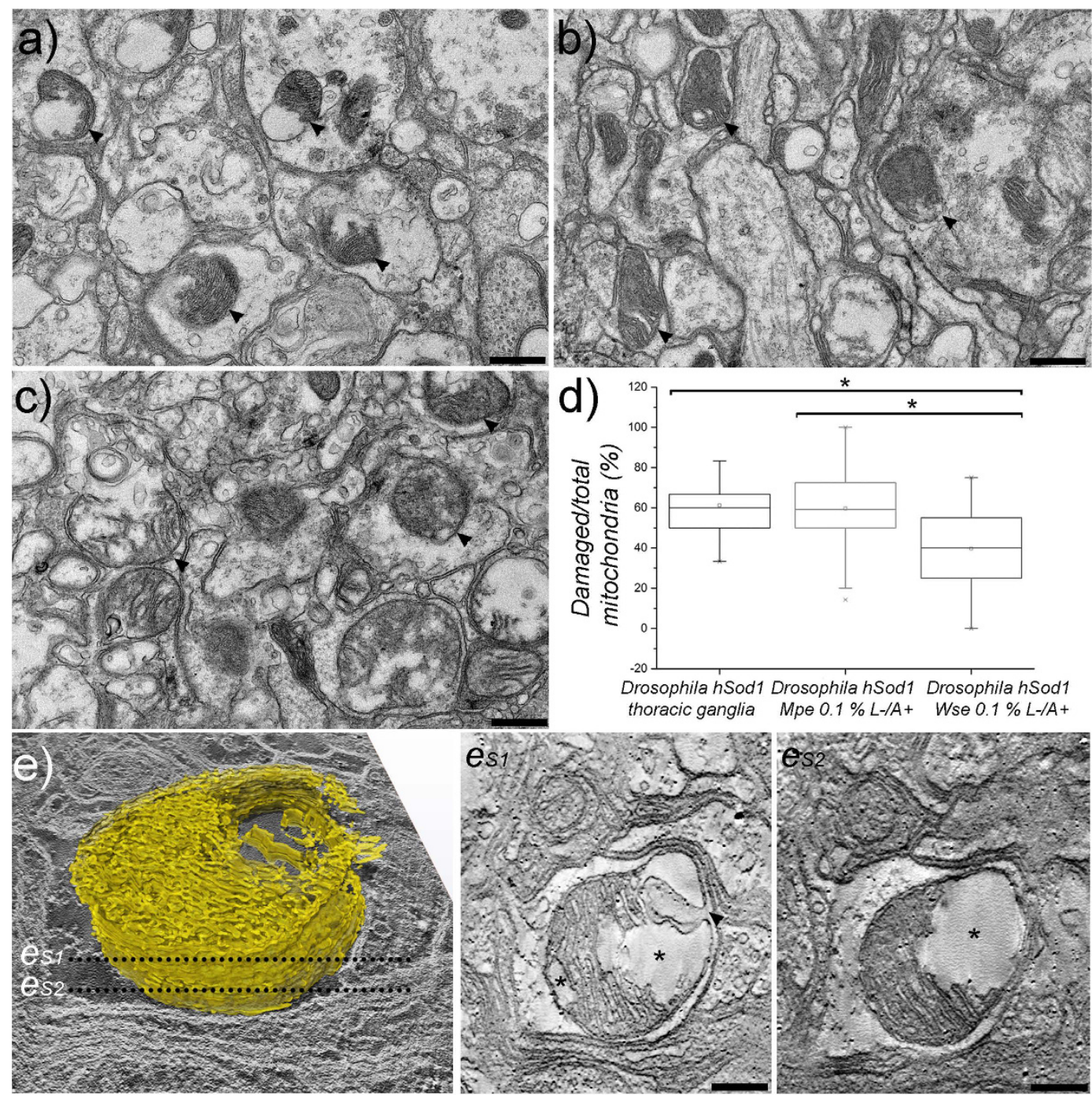

Figure 6. Electron microscopy. Representative TEM images of thoracic ganglia (T1-T2 regions) in Drosophila hSOD1 untreated (a) and treated with $W s e \mathrm{~L}^{-} / \mathrm{A}^{+}(\mathbf{b})$ and $M p e \mathrm{~L}^{-} / \mathrm{A}^{+}$(c) respectively. (d) Percentage of damaged versus total mitochondria in thoracic ganglia (T1-T2 regions) of Drosophila hSOD1 untreated (hSOD1, $\mathrm{n}$ mitochondria $=464$ ) and treated with a $0.1 \%$ solution of Mucuna (hSOD1 Mpe, $\mathrm{n}$ mitochondria $=524$ ) and Whitania (hSOD1 Wse, n mitochondria $=271$ ) respectively. ${ }^{\star}$ t-Student test $\mathrm{p}$-value $<0.01$. (e) $3 \mathrm{D}$ model representing the reconstruction of a single mitochondrion in Drosophila hSOD1 thoracic ganglia (T1-T2 regions). The $3 \mathrm{D}$ model is set on a tomographic section. The es 1 and es2 images are digitally inverted single tomographic slices corresponding to sections es 1 and es 2 in the 3D reconstruction. Arrowheads point to mitochondria. Asterisks point to damaged areas in the mitochondrion (see main text). Scale bars are $0.4 \mu \mathrm{m}$ in $(\mathbf{a}, \mathbf{b})$ and $(\mathbf{c})$, and $0.2 \mu \mathrm{m}$ in es 1 and es2.

from presynaptic terminals. Our findings suggest that the effects of Wse and Mpe on the functional changes associated with the hSOD1 over expression seem clearly connected to beneficial aspects of these treatments. Furthermore, hSOD1 flies showed an increase in failure of PSPs after increasing frequency stimulation compared with GAL4 flies. Even partially different from other reports ${ }^{21}$, that showed such modification later in life, our data are strongly consistent with climbing attitude results that show an impairment that appear earlier in life. In addition, we found that both Wse and Mpe were effective also in reverting the changes on train frequency responsiveness observed in hSOD1 flies. Interestingly, the effect of Wse on genetically-mediated impairments on percentage of failures at increasing frequency stimulation is in agreement with our previous data on LRRK2 mutants ${ }^{19}$. Furthermore, the results on the percentage of failures following Mpe treatment are in agreement with the morphological observations of our previous study made with PINK1 ${ }^{\mathrm{B} 9}$ PD mutants ${ }^{18}$ in which we observed an increased expression of $\mathrm{T}$ bars. These observations point to the ability of these extracts to overall impact, perhaps through different mechanisms, on impaired release of neurotransmitters from pre-synaptic terminals.

Locomotor impairment as well as altered muscle contraction here presented well correlate with the presence of a high density of damaged mitochondria in the hSOD1 T1-T2 regions of thoracic ganglia. Boillée et al. ${ }^{2}$ by means of in vivo studies, emphasizes the importance of the glia as well as the glia-neurons interaction in the development 
of ALS. Although on the basis of our results we cannot exclude an involvement of glia or specific glia-neuron interactions in ALS, our EM analysis showed that mainly thoracic ganglia neurons, characterized by the presence of T-Bars in their presynaptic terminals, showed damaged mitochondria. The damaged mitochondria at level of presynaptic terminals may be in agreement with electrophysiological data showing that hSOD1 overexpression is also related to an impaired postsynaptic response to high frequency stimulation of presynaptic terminals that fired to DLM motoneurons.

In agreement with other studies ${ }^{33-38}$ our results point to a possible link between SOD1, mitochondrial dysfunction and ALS. Intriguingly, we showed that the over-expression of the wild type hSOD1 also causes mitochondrial alterations. Thus, not only mutation in hSOD1 but also alteration in its expression may lead to mitochondrial dysfunction and possibly drive to ALS symptoms. Interestingly it has been shown that overexpressed mutant SOD1 mislocalize with mitochondria affecting their functions and contributing to the degeneration of motoneurons leading to $\mathrm{ALS}^{38,39}$. Although the proposed model is appealing, further experiments are needed to clarify the mechanisms by which wild type hSOD1 overexpression affects mitochondria functioning. We hypothesize that an overexpression of human SOD1 in the motoneurons could be regarded as a destabilizing solution condition ${ }^{27}$ and mitochondria are altered as a consequence.

Treating the hSOD1 mutants with Wse was able to significantly decrease the number of damaged mitochondria in T1-T2 thoracic ganglia region rescuing also climbing as well as muscle electrophysiological parameters. Intriguingly, Wse exhibited a similar rescue effect on damaged mitochondria in LRRK2 loss-of-function Drosophila model of $\mathrm{PD}^{19}$. The use of this drug as a therapy is really promising for many pathologies, but no data are available specifically on Withania somnifera extract against ALS, even if a number of papers are present regarding the effects of active molecules present in the extract according to the widely accepted anti-oxidant activity (glycowithanolides sitoindosides VII-X and withaferin A) ${ }^{32,40-42}$. More recently, Patel et al. ${ }^{43}$ reported that Withaferin A reduces the levels of misfolded SOD1 in a mouse model of ALS. Instead, the present work specifically used Withania somnifera and Mucuna pruriens extracts against ALS-SOD1. Moreover, the present work indicates that Drosophila melanogaster is a powerful model of ALS, convenient because of the reproducibility, the low cost and a relatively short life-span with respect to other models. More importantly, it indicates that this organism can be used as a model in studying phytoterapic approaches to ALS. Specifically Withania somnifera extract presents, compared to the single active molecules above reported, different advantageous being more safety, as it is used in Ayurvedic medicine since many centuries, cheaper as it does not need extraction costs and easy to be administered. As already cited, Withania somnifera powder is considered very safe for values ranging from $0.001 \mathrm{mg}$ to $1000 \mathrm{mg}$ per $\mathrm{kg}$. of body weight, showing modest tranquilizers and hypotensive effects at $25 \mathrm{mg} / \mathrm{kg}^{32}$.

Additional pre-clinical studies are necessary in order to verify the efficacy of this drugs on ALS-TDP43 while the present data strongly support the use of this extract to counteract the ALS-symptoms in humans. In this regard, we acknowledge that clinical studies will be required in order to characterize the optimal conditions (dosage and duration) at which these drugs might be successfully used to counteract this terrible disease.

In this respect, the observation that the administration of Wse and $M p e$ to flies as $\mathrm{L}^{+} / \mathrm{A}^{+}$induces a further reduction of lifespan as compared to WT controls and untreated hSOD1 indicates that these phytoterapics may exert their effects -as a drug-following a hormesis-like dose-response curve ${ }^{44}$, similarly to what observed in the LRRK $^{\mathrm{WD} 40}$ Drosophila PD mutant ${ }^{19}$, and further highlights the need to assess the proper concentration and duration of treatment.

Unlike the Wse treatment, the Mpe one on Drosophila overexpressing hSOD1 did not rescue mitochondrial impairment in the thoracic ganglia. This result is surprising since the same treatment well rescued climbing as well as muscle electrophysiological parameters. Moreover, $M p e$ well restored mitochondrial impairment in the olfactory bulbs of PD-PINK ${ }^{\mathrm{B} 9}$ mutants ${ }^{17}$. These results, that need further experiments to be fully understood, suggest that Wse and Mpe rescue mitochondrial damage probably through different molecular mechanisms. This is suggested also by the significantly different quantitative composition of Mpe that includes, among others, a number of anti-oxidant agents such as $\mathrm{Fe}^{2+}$ and glutathione responsible of preventing the consequences of both hSOD1 over-expression as well as mitochondrial functional defects due to silencing of PINK1 ${ }^{18}$. In addition several evidences suggest that a common path in different neurodegenerative desease is the increase in the formation of inflammatory markers such as pro-inflammatory cytokines including interleukin- 6 and tumor necrosis factor- $\alpha$ , nitric oxide and reactive oxygen species (ROS) (see D'Ambrosi et al. ${ }^{45}$ for review) contributing to mitochondrial function and inducing cell death. In this regard, it has widely reported that Wse show a potent inhibitory effect on inflammatory markers such as ROS in a mouse model of lupus ${ }^{46,47}$. Interestingly $W s e$ reduced rotenone-induced oxidative impairment and mitochondrial respiratory chain enzymes and such impairments were responsible for reduced locomotor deficits and lethality in a Drosophila melanogaster model of Parkinson induced by rotenone, a naturally occurring common pesticide which specifically inhibits mitochondrial complex-I activity ${ }^{48}$. We here speculate that Wse as well as Mpe may affect the activity of mitochondrial complex-I inhibiting the negative action induced by SOD1 mutation onto mitochondrial function.

Taken together, our results demonstrate that an overexpression of wild hSOD1, in Drosophila melanogaster flies, is able to alter the motor coordination of mutant animals, an effect that is already present in young animals and remains even in adulthood. The positive effect of Wse as well as Mpe, results in an improved performance (climbing) and in a recover of normal electrophysiological function of locomotor system. Moreover, on the basis of our results it seems clear that both Parkinson Disease (PD) and Amyotrophic Lateral Sclerosis share mitochondrial dysfunction even if they differ in both genetic and metabolic pathways. Finally, given the role of inflammation in the development of ALS, the known anti-inflammatory properties of these extracts and the viability of their clinical use, these results suggest that the application of Wse and, to lesser extent, of Mpe might represent a valuable pharmacological strategy to counteract the progression of ALS and related symptoms. 


\section{Methods}

Flies. We used the GAL4-UAS binary system ${ }^{49,50}$ to over-express human SOD1 (hSOD1) specifically in motoneuron cells of $D$. melanogaster. For these experiments, we crossed GAL4D42 (dSOD1, \#42737; from now on reported as GAL4) with UAS-hSOD1 (hSOD1, \#33656, from Bloomington Stock Center; Fly Base: http://flybase. bio.indiana.edu) flies. After emergence from pupae, GAL4 (isogenic controls) and hSOD1 mutant male flies were reared on a standard cornmeal-yeast-agar medium in controlled environmental conditions $\left(24-25^{\circ} \mathrm{C}\right.$; $60 \%$ relative humidity; light/dark =12/12 hours).

In detail, a part of GAL4 and hSOD1 mutants, four groups of mutant flies were reared on a standard medium supplemented with Withania somnifera (Wse) or Mucuna pruriens (Mpe) extracts (kindly provided by Natural Remedies Pvt. Ltd., Bangalore, India). In agreement with our previous studies ${ }^{18,19}$ hSOD1 mutants were supplied with Wse or Mpe at $0.1 \%$ w/w concentrations both as larvae and adults $\left(\mathrm{L}^{+} / \mathrm{A}^{+}\right)$or as adults only $\left(\mathrm{L}^{-} / \mathrm{A}^{+}\right)$. Moreover, lifespan of GAL4 Wse- and Mpe-treated flies as $\mathrm{L}^{-} / \mathrm{A}^{+}$was analysed.

The effects of Wse or Mpe were assayed at different age steps (I: 3-6; II: 10-15; III: 20-25 days old). The experiments on life span, using different time of administration at the same concentrations of Wse or Mpe (see below in Survival curves) provided the basis for selecting the time of drug administration at which conduct the behavioural, electrophysiological and morphological experiments. In particular, based on lifespan results, behavioural, electrophysiological and transmission electron microscopy (TEM) were restricted to group II flies after $0.1 \% \mathrm{w} / \mathrm{w}$ Wse or Mpe administration as $\mathrm{L}^{-} / \mathrm{A}^{+}$. Standard genetic procedures were used during the study.

Survival curves. As in Drosophila mutants for Parkinson's disease both Wse and Mpe showed effects dependent on the duration of the treatment; Wse or Mpe standardized methanolic extracts were administered in two different modalities: as adults $\left(\mathrm{L}^{-} / \mathrm{A}^{+}\right)$only or as larvae and adults $\left(\mathrm{L}^{+} / \mathrm{A}^{+}\right)$with the aim of selecting the optimal Mpe and Wse administration time. In detail, flies were grown on standard diet supplemented with $0.1 \%$ w/w concentrations of Wse or Mpe. Cohorts of 60 flies (10 flies/tube) from each experimental group (i.e. GAL4, Wse-untreated, Wse-treated hSOD1, Mpe-untreated and Mpe-treated hSOD1), were monitored every 2 days for their survival. Mortality was analyzed using Kaplan-Meier survival curves and the statistical comparisons were made with a Gehan-Breslow-Wilcoxon test. All experiments were done in triplicate. All experiments were done in triplicate, except for Wse- and Mpe-treated Gal4 flies, that were done in duplicate.

Climbing assay. The climbing assay (negative geotaxis assay) was used to assess locomotor ability both in GAL4 control flies and in the hSOD1 mutant ones as already reported by Liu et al. ${ }^{51}$, Poddighe et al. ${ }^{17,18}$ and De Rose et al. ${ }^{19}$.

Climbing data were obtained from different age groups (I: 3-6; II: 10-15; III: 20-25 days old) of untreated-GAL4, Wse-untreated, Wse-treated, Mpe-treated and Mpe-untreated hSOD1 mutants. Cohorts of 60 flies from each experimental group were subjected to the assay. Flies were placed individually in a vertically-positioned plastic tube (length $10 \mathrm{~cm}$; diameter $1.5 \mathrm{~cm}$ ) and tapped to the bottom. Climbing time (seconds) was recorded upon crossing a line drawn at $6 \mathrm{~cm}$ from the bottom. The number of flies that could climb unto, or above, this line within 10 seconds was recorded and expressed as percentage of total flies. Data were expressed as average \pm standard error of the mean (SEM) from three experiment replications. Statistically significant differences $(\mathrm{p}<0.05)$ were analyzed between GAL4 vs. hSOD1 and between untreated-hSOD1 vs. treated ones by means of the one-way ANOVA followed by HSD post-hoc test.

Electrophysiological recordings. At the time of experiments, flies from the different experimental groups at age step II (10-15 days) were anesthetized by using CO2 and tightly anchored to a wax support with ventral side down, as previously reported ${ }^{30}$, and visualized under a stereomicroscope. In order to activate the GFS of the fly, two tungsten stimulating electrodes, connected to a stimulator (Master 8, A.M.P.I, Jerusalem, IL, USA) triggered by a stimulus isolation unit (DS2A, Digitimer Ltd., Hertfordshire UK), were placed into both eyes of the fly. Stimulus intensity was increased until the postsynaptic potential response was observed and maximal stimulation intensity was not greater than $10 \mathrm{~V}$. Moreover the range of stimulation was very wide (the positive artefact, that represent the stimulation intensity, is perceptible before every single) and no correlation between stimulation intensity and type of animal tested was observed (data not shown). A ground tungsten wire was placed into the fly abdomen. A borosilicate recording electrode, shaped by a horizontal puller (P97, Fleming Brown, Sutter Instruments, Novato, CA, USA) with a resistance of $4-5 \mathrm{M} \Omega$ when filled with $3 \mathrm{M} \mathrm{KCl}$, was placed into the right or left backside of the fly along the $45 \mathrm{a}$ and $45 \mathrm{~b}$ fibres of the Dorsal Longitudinal Muscle fibres (DLMs). Evoked post-synaptic potentials (PSPs) were recorded with an Axopatch 2-B amplifier (Axon Instruments, Foster City, $\mathrm{CA}$ ), filtered at $0.5 \mathrm{kHz}$ and digitized at $1 \mathrm{kHz}$. PSPs were recorded in bridge mode, measured using peak and event detection software pCLAMP 8.2 (Axon Instruments, Foster City, CA), and analyzed off-line by pCLAMP fit software (Axon Instruments, Foster City, CA). All recordings were obtained from at least 10 different flies belonging to each experimental group. Experiments were blind to the treatment. Electrophysiological experiments were performed by applying a protocol consisting in a single GFS stimulation, delivered every $20 \mathrm{~s}$, followed by PSP recording. The "frequency of following" was determined by delivering trains of 10 stimuli at increasing frequencies (from 10 to $200 \mathrm{~Hz}$ ) and the failures, as the percentage of lacking responses at each train, were calculated. As previously published ${ }^{19}$ we also evaluated the amplitude (peak of the PSP expressed in $\mathrm{mV}$ ) as well as the latency (interval between the stimulating artifact and the time at PSP peak, expressed in ms) of the first PSP evoked by the $10 \mathrm{~Hz}$ stimulation train. Data are expressed as mean +S.E.M. and analyzed by one or two-way ANOVAs followed by Bonferroni’s post-hoc tests.

Electron microscopy analysis. Untreated hSOD1, Wse- and Mpe-treated hSOD1 mutants were anesthetized using $\mathrm{CO} 2$ and carefully decapitated. Once rapidly removed, the thoracic ganglia were fixed in a mixture of 
$2 \%$ glutaraldehyde and $2 \%$ paraformaldehyde in $0.1 \mathrm{M}$ cacodylate buffer, washed several times in the same buffer, post-fixed in $1 \%$ osmium tetroxide in distilled water for 2 hours, and stained overnight at $4{ }^{\circ} \mathrm{C}$ in an aqueous $0.5 \%$ uranyl acetate solution. After several washes in distilled water, the samples were dehydrated in a graded ethanol series, and embedded in SPURR resin. Semi-thin coronal sections of the whole ganglion were cut with a Leica EM UC6 ultramicrotome, stained with toluidine blue and observed with a Leica DM2700 P light microscope. Sections of about $70 \mathrm{~nm}$ corresponding to portions of the thoracic ganglia were cut with a diamond knife on a Leica EM UC6 ultramicrotome. Transmission electron microscopy (TEM) images were collected with a FEI Tecnai G2 F20 (FEI Company, The Netherlands) and a Jeol JEM 1011 (Jeol, Japan) electron microscopes and recorded with a 1 and $2 \mathrm{Mp}$ charge-coupled device (CCD) camera (Gatan BM Ultrascan and Gatan Orius SC100, respectively). The number of damaged mitochondria within the thoracic ganglia $\mathrm{T} 1$ and $\mathrm{T} 2$ regions (expressed as percentage of the total number of mitochondria/sampled area) was evaluated in untreated hSOD1, Wse-and-Mpe-treated hSOD1 mutants. More than 2300 mitochondria were randomly sampled on 227 non-overlapping micrographs at a final magnification of $6000 \mathrm{x}$, corresponding to a total sampled area of more than $2800 \mu^{2}$. Damaged mitochondria were recognized for the presence of swollen external membrane, clearly fragmented cristae and inhomogeneous electron transparent mitochondrial matrix. The mean differences were tested using a two-tailed t-test and a $\mathrm{p}<0.01$ level was considered statistically significant. EM tomography was performed in scanning TEM (STEM) using a high angular annular dark field (HAADF) detector on $400 \mathrm{~nm}$ sections of Drosophila hSOD1 the thoracic ganglia T1-T2 region. The tilt series were acquired from \pm 65 degree tilt range using a Saxton tilt scheme with 3 -degree increment at 0 tilt. A final magnification of $28000 \mathrm{x}$ was used, corresponding to a pixel size of $3.6 \mathrm{~nm}$. Computation of tomograms was done with the IMOD (version 4.8.40) software package ${ }^{52}$. Isosurface based segmentation and three-dimensional visualization on unbinned and unfiltered tomograms were performed using the Amira software package (FEI Visualization Science Group, Bordeaux, France).

\section{References}

1. Rosen, D. R. et al. Mutations in Cu/Zn superoxide dismutase gene are associated with familial amyotrophic lateral sclerosis. Nature 362, 59-62 (1993).

2. Boillée, S., Vande Velde, C. \& Cleveland, D. ALS: A Disease of Motor Neurons and Their Nonneuronal Neighbors. Neuron 52, 39-59 (2006).

3. Bruijn, L. I. et al. ALS-linked SOD1 mutant G85R mediates damage to astrocytes and promotes rapidly progressive disease with SOD1-containing inclusions. Neuron 18, 327-338 (1997).

4. Gurney, M. E. et al. Motor neuron degeneration in mice that express a human $\mathrm{Cu}, \mathrm{Zn}$ superoxide dismutase mutation. Science (80-. ). 264, 1772-1775 (1994).

5. Wong, P. C. et al. An adverse property of a familial ALS-linked SOD1 mutation causes motor neuron disease characterized by vacuolar degeneration of mitochondria. Neuron 14, 1105-1116 (1995).

6. Ransohoff, R. M. How neuroinflammation contributes to neurodegeneration. Science 353, 777-83 (2016).

7. Bruijn, L. I., Miller, T. M. \& Cleveland, W. Unraveling the mechanisms involved in motor neuron degeneration in ALS. Annu. Rev. Neurosci 27, 723-49 (2004).

8. Ciechanover, A. \& Kwon, Y. T. Degradation of misfolded proteins in neurodegenerative diseases: therapeutic targets and strategies. Exp. Mol. Med. 47, e147 (2015).

9. Silverman, J. M. et al. Disease Mechanisms in ALS: Misfolded SOD1 Transferred Through Exosome-Dependent and ExosomeIndependent Pathways. Cellular and Molecular Neurobiology 36, 377-381 (2016).

10. Lee, S. \& Kim, H.-J. Prion-like Mechanism in Amyotrophic Lateral Sclerosis: are Protein Aggregates the Key? Exp. Neurobiol. 24, 1-7 (2015).

11. Lu, B. \& Vogel, H. Drosophila Models of Neurodegenerative Diseases. Annu. Rev. Pathol. Mech. Dis. 4, 315-342 (2009).

12. Greeve, I. et al. Age-dependent neurodegeneration and Alzheimer-amyloid plaque formation in transgenic Drosophila. J. Neurosci. 24, 3899-906 (2004).

13. Iijima, K. et al. Dissecting the pathological effects of human A $\beta 40$ and A $\beta 42$ in Drosophila: A potential model for Alzheimer's disease. Proc. Natl. Acad. Sci. USA 101, 6623-6628 (2004).

14. Feany, M. B. \& Bender, W. W. A Drosophila model of Parkinson's disease. Nature 404, 394-8 (2000).

15. Greene, J. C. et al. Mitochondrial pathology and apoptotic muscle degeneration in Drosophila parkin mutants. Proc. Natl. Acad. Sci. USA 100, 4078-83 (2003).

16. Yang, Y., Nishimura, I., Imai, Y., Takahashi, R. \& Lu, B. Parkin suppresses dopaminergic neuron-selective neurotoxicity induced by Pael-R in Drosophila. Neuron 37, 911-924 (2003).

17. Poddighe, S. et al. Impaired Sense of Smell in a Drosophila Parkinson's Model. PLoS One 8 (2013).

18. Poddighe, S. et al. Mucuna pruriens(Velvet bean) rescues motor, olfactory, mitochondrial and synaptic impairment in PINK1B9 Drosophila melanogaster genetic model of Parkinson's disease. PLoS One 9 (2014).

19. De Rose, F. et al. Functional and Morphological Correlates in the Drosophila LRRK2 loss-of-function Model of Parkinson's Disease: Drug Effects of Withania somnifera (Dunal) Administration. PLoS One 11, e0146140 (2016).

20. Mockett, R. J., Radyuk, S. N., Benes, J. J., Orr, W. C. \& Sohal, R. S. Phenotypic effects of familial amyotrophic lateral sclerosis mutant Sod alleles in transgenic Drosophila. Proc. Natl. Acad. Sci. USA 100, 301-306 (2003).

21. Watson, M. R., Lagow, R. D., Xu, K., Zhang, B. \& Bonini, N. M. A drosophila model for amyotrophic lateral sclerosis reveals motor neuron damage by human SOD1. J. Biol. Chem. 283, 24972-81 (2008).

22. Katzenschlager, R. et al. Mucuna pruriens in Parkinson's disease: a double blind clinical and pharmacological study. J. Neurol. Neurosurg. Psychiatry 75, 1672-1677 (2004).

23. Lieu, C. A. et al. The antiparkinsonian and antidyskinetic mechanisms of Mucuna pruriens in the MPTP-treated nonhuman primate. Evidence-based Complement. Altern. Med. 2012 (2012).

24. Kasture, S., Mohan, M. \& Kasture, V. Mucuna pruriens seeds in treatment of Parkinson's disease: Pharmacological review. Orient. Pharm. Exp. Med. 13, 165-174 (2013).

25. Sala Frigerio, C. \& De Strooper, B. Alzheimer's Disease Mechanisms and Emerging Roads to Novel Therapeutics. Annu. Rev. Neurosci. 39, 57-79 (2016).

26. Browne, E. C. \& Abbott, B. M. Recent progress towards an effective treatment of amyotrophic lateral sclerosis using the SOD1 mouse model in a preclinical setting. European Journal of Medicinal Chemistry 121, 918-925 (2016).

27. Stathopulos, P. B. et al. Cu/Zn superoxide dismutase mutants associated with amyotrophic lateral sclerosis show enhanced formation of aggregates in vitro. Proc. Natl. Acad. Sci. 100, 7021-7026 (2003).

28. RajaSankar, S., Manivasagam, T. \& Surendran, S. Ashwagandha leaf extract: A potential agent in treating oxidative damage and physiological abnormalities seen in a mouse model of Parkinson's disease. Neurosci. Lett. 454, 11-15 (2009). 
29. Gupta, M. \& Kaur, G. Aqueous extract from the Withania somnifera leaves as a potential anti-neuroinflammatory agent: a mechanistic study. J. Neuroinflammation 13, 193 (2016).

30. Augustin, H., Allen, M. J. \& Partridge, L. Electrophysiological recordings from the giant fiber pathway of D. melanogaster. J. Vis. Exp. 1-5, doi: 10.3791/2412 (2011).

31. Casci, I. \& Pandey, U. B. A fruitful endeavor: Modeling ALS in the fruit fly. Brain Res. 1607, 47-74 (2015).

32. Mishra, L. C., Singh, B. B. \& Dagenais, S. Scientific basis for the therapeutic use of Withania somnifera (ashwagandha): A review. Alternative Medicine Review 5, 334-346 (2000).

33. Le Verche, V. \& Przedborski, S. Is amyotrophic lateral sclerosis a mitochondrial channelopathy? Neuron 67, 523-524 (2010).

34. Bahadorani, S. et al. Expression of zinc-deficient human superoxide dismutase in Drosophila neurons produces a locomotor defect linked to mitochondrial dysfunction. Neurobiol. Aging 34, 2322-2330 (2013).

35. Ferri, A. et al. Familial ALS-superoxide dismutases associate with mitochondria and shift their redox potentials. Proc. Natl. Acad. Sci. USA 103, 13860-13865 (2006).

36. Liu, J. et al. Toxicity of familial ALS-linked SOD1 mutants from selective recruitment to spinal mitochondria. Neuron 43, 5-17 (2004).

37. Pasinelli, P. et al. Amyotrophic lateral sclerosis-associated SOD1 mutant proteins bind and aggregate with Bcl-2 in spinal cord mitochondria. Neuron 43, 19-30 (2004)

38. Tan, W., Pasinelli, P. \& Trotti, D. Role of mitochondria in mutant SOD1 linked amyotrophic lateral sclerosis. Biochim. Biophys. Acta 1842, 1295-301 (2014).

39. Pasinelli, P. \& Brown, R. H. Molecular biology of amyotrophic lateral sclerosis: insights from genetics. Nat. Rev. Neurosci. 7, 710-723 (2006).

40. Alam, N. et al. High catechin concentrations detected in Withania somnifera (ashwagandha) by high performance liquid chromatography analysis. BMC Complement. Altern. Med. 11, 65 (2011).

41. Kaileh, M. et al. Withaferin A strongly elicits I kappa B kinase beta hyperphosphorylation concomitant with potent inhibition of its kinase activity. J. Biol. Chem. 282, 4253-4264 (2007).

42. Kuboyama, T., Tohda, C. \& Komatsu, K. Effects of Ashwagandha (roots of Withania somnifera) on neurodegenerative diseases. Biol. Pharm. Bull. 37, 892-7 (2014).

43. Patel, P., Julien, J. P. \& Kriz, J. Early-Stage Treatment with Withaferin A Reduces Levels of Misfolded Superoxide Dismutase 1 and Extends Lifespan in a Mouse Model of Amyotrophic Lateral Sclerosis. Neurotherapeutics 12, 217-233 (2015).

44. Mattson, M. P. Hormesis defined. Ageing Research Reviews 7, 1-7 (2008).

45. D’Ambrosi, N., Rossi, S., Gerbino, V. \& Cozzolino, M. Rac1 at the crossroad of actin dynamics and neuroinflammation in Amyotrophic Lateral Sclerosis. Front. Cell. Neurosci. 8, 279 (2014).

46. Minhas, U., Minz, R. \& Bhatnagar, a. Prophylactic effect of Withania somnifera on inflammation in a non-autoimmune prone murine model of lupus. Drug Discov Ther 5, 195-201 (2011).

47. Minhas, U., Minz, R., Das, P. \& Bhatnagar, A. Therapeutic effect of withania somnifera on pristane-induced model of SLE. Inflammopharmacology 20, 195-215 (2012).

48. Manjunath, M. J. \& Muralidhara. Standardized extract of Withania somnifera (Ashwagandha) markedly offsets rotenone-induced locomotor deficits, oxidative impairments and neurotoxicity in Drosophila melanogaster. J. Food Sci. Technol. 52, 1971-1981 (2015).

49. Brand, A. H. \& Perrimon, N. Targeted gene expression as a means of altering cell fates and generating dominant phenotypes. Development 118, 401-15 (1993).

50. Fischer, J. a, Giniger, E., Maniatis, T. \& Ptashne, M. GAL4 activates transcription in Drosophila. Nature 332, 853-856 (1988).

51. Liu, Z. et al. A Drosophila model for LRRK2-linked parkinsonism. Proc. Natl. Acad. Sci. USA 105, 2693-2698 (2008).

52. Kremer, J. R., Mastronarde, D. N. \& McIntosh, J. R. Computer visualization of three-dimensional image data using IMOD. J. Struct. Biol. 116, 71-6 (1996)

\section{Acknowledgements}

We would like to thank Annacarla Arru, Giuliana Colella and Ignazio Collu to take care the flies. We thank Ameya Kasture for helping in the manuscript revision and many suggestions for discussion section. This work was supported by Rotary Club Sanluri to F.M. and G.B.

\section{Author Contributions}

F.DeR., R.M., G.T. and A.L., conceived and designed the experiments. F.DeR., R.M., G.T. and T.C., performed the experiments. A.L., F.DeR., P.S., G.T., S.P., E.A., R.M. and E.S., analyzed the data. A.L., S.K., E.A., R.M., E.S., G.B. and F.M., contributed reagents/materials/analysis tools. E.A., S.K., P.S., G.T., R.M. and A.L., contributed to the interpretation of the data and wrote the manuscript. All the Authors reviewed and approved the manuscript.

\section{Additional Information}

Supplementary information accompanies this paper at http://www.nature.com/srep

Competing financial interests: The authors declare no competing financial interests.

How to cite this article: De Rose, F. et al. Differential effects of phytotherapic preparations in the hSOD1 Drosophila melanogaster model of ALS. Sci. Rep. 7, 41059; doi: 10.1038/srep41059 (2017).

Publisher's note: Springer Nature remains neutral with regard to jurisdictional claims in published maps and institutional affiliations.

(c) (i) This work is licensed under a Creative Commons Attribution 4.0 International License. The images or other third party material in this article are included in the article's Creative Commons license, unless indicated otherwise in the credit line; if the material is not included under the Creative Commons license, users will need to obtain permission from the license holder to reproduce the material. To view a copy of this license, visit http://creativecommons.org/licenses/by/4.0/

(C) The Author(s) 2017 